\title{
Analysis on Calculation of Transverse Swing Amount of Mine Hoisting Container under Flexible Constrains
}

\author{
Kai Feng ${ }^{1}$, Yujun Xue ${ }^{1,2}$, Fang Yang ${ }^{2}$, Jishun $\mathrm{Li}^{2}$, Wei $\mathrm{Ma}^{1,2}$ \\ ${ }^{1}$ School of Mechatronics Engineering, Henan University of Science and Technology, Luoyang \\ 471003; \\ ${ }^{2}$ Henan Key Laboratory for Machinery Design and Transmission System, Henan University of \\ Science and Technology, Luoyang 471003
}

\begin{abstract}
Increasing transverse swing of container has a direct impact on safety of multi-rope friction mine hoist. Under the strain of flexible guide, increasing transverse swing is related with parameters of mine hoisting system. Take hoist container under strain of steel rope guide as the research object, considers increasing hoist speed of hoist container, increasing load of steel rope terminal and lateral stiffness of steel rope guide and other parameters to establish a solving equation of increasing swing amount of container, and then analyze transverse swing amount of hoist container under different parameters of tension of guide rope, hoist speed and load on the terminal of hoist steel rope. Research shows: lateral stiffness of guide rope is inversely proportional to transverse swing amount of hoist container under flexible strains; hoisting speed and increasing load of steel rope terminal have greater effect on increasing swing amount of container in the middle segment of guide than in the starting and ending point.
\end{abstract}

Keywords: Flexible cage; Upgrade container; Lateral stiffness; Lateral runout

\section{柔性约束下矿井提升容器的横向摆动量计算分析}

$$
\text { 冯凯 }{ }^{1} \text {, 薛玉君 }{ }^{1,2} \text {, 杨芳 }{ }^{2}, \text { 李济顺 }^{2} \text {, 马伟 }{ }^{1,2}
$$

(1. 河南科技大学 机电工程学院 河南洛阳 471003; 2. 河南科技大学 河南省机械设计及传动系统重点 实验室 河南洛阳 471003)

摘要: 提升容器横向偏摆直接影响多绳摩擦矿井提升机的安全。柔性罐道约束下, 提升容器横向摆动与矿井提升系统的 参数有关。以钢丝绳罐道约束下提升容器为研究对象, 考虑提升容器的提升速度、提升钢丝绳终端载荷和钢丝绳罐道横向刚 度等参数建立提升容器偏摆量求解方程, 分析提升容器在不同罐道绳张紧力、提升速度、提升钢丝绳终端载荷等参数下的横 向偏摆量。研究表明: 柔性约束下罐道绳的横向刚度与提升容器的横向偏摆量存在反比例关系; 提升速度和提升钢丝绳终端 载荷在罐道绳中段对提升容器偏摆量影响较大, 而在起始和终点段影响较小。

关键词: 柔性罐道; 提升容器; 横向刚度; 横向偏摆量

中图分类号：TH16 文献标示：A

\section{引言}

提升容器是矿井提升装备的重要组成部分。正常运行过程中, 由于提升钢丝绳自身的开捻力、科氏力、 提升容器相互交错时气流的扰动都会使提升容器和提升钢丝绳产生明显的横向偏摆。为此, 国内外许多专 家都对矿井提升系统提升端的横向振动做了相关研究。包继虎 [1]等通过建立曳引电梯提升系统的横振模 型, 分析了提升钢丝绳在天轮出有无激励作用下的振动情况。吴娟 [2] 等应用 Hamilton 原理建立多绳提升 
系统变长度提升钢丝绳横向振动模型, 仿真分析处不同运行阶段提升钢丝绳的横向振动规律以及刚度、阻 尼对横向振动的影响; 刘义 [3] 等建立了提升钢丝绳和刚性罐道系统动力学模型, 对提升系统的横向振动 特性进行了研究。Kaczmarczyk S[4-5]建立了连续化钢丝绳三维振动模型, 对重载深井进行了分析。李玉 瑾 [6]建立了摩擦提升系统的二、三自由度分析模型, 研究了梯形加速度变化对提升钢丝绳上张力的响应 特性。曹国华 $[7]$ 等建立了基于分布参数的连续模型的提升钢丝绳在装载过程冲击载荷下的纵向一扭转耦 合振动数学模型, 研究提升钢丝绳在箕斗装载、承接、荷载进罐等振动状况。潘英 [8]等通过建立钢丝绳 弹性连续模型, 假定钢丝绳长度不变情况狂下矩形、梯形、正弦形等加速度对钢丝绳动张力响应的特性。 Ostapenko [9]研究了提升钢丝绳的变长度问题, 并给了钢丝绳纵向振动的精确解解析。

通过以上研究可知, 国内外研究的重点都是对刚性罐道下提升钢丝绳的横向振动和纵向振动的研究, 以及提升钢丝绳上动张力的研究。但是, 目前关于柔性约束下矿井提升机提升容器横向偏摆量计算分析的 研究很少见到报道。相比于刚性罐道, 钢丝绳是柔性的, 提升容器在水平方向更容易摆动, 如果提升容器 水平摆动过大，极易在交汇处会发生撞击事故。

本文以钢丝绳罐道约束下提升容器为研究对象, 建立柔性约束下提升容器横向偏摆量计算公式, 研究 罐道绳张紧力、提升容器提升速度、提升钢丝绳终端载荷等参数对提升容器偏摆量的影响, 获得不同参数 下矿井提升容器偏摆量随提升高度的变化特性，为矿井提升系统的设计提供依据。

\section{1 多绳提升容器的摆动轨迹分析}

多绳提升容器的摆动形式主要是波浪形摆动, 它的摆动轨迹可以看做周期和峰值不同的正弦曲线 [10], 如图 1 所示。这种摆动轨迹是由于上升过程中提升容器受到提升钢丝绳、地球自转和井筒内气流等 因素对提升容器的横向作用力以及钢丝绳罐道对提升容器的恢复反作用力造成的。可以看出提升容器的最 大摆动量在提升高度的中间位置附近, 提升容器摆动行为当做简谐振动。

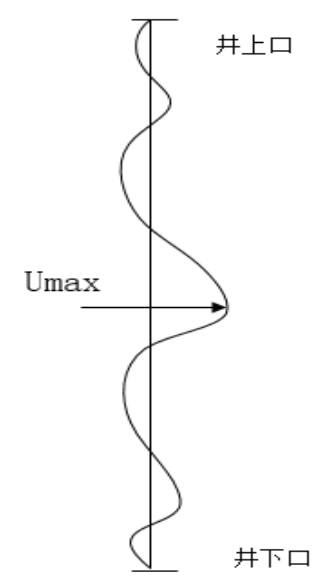

图 1 多绳提升系统提升容器摆动轨迹

根据简谐振动周期公式可以得到提升容器的摆动周期为:

$$
T=2 \pi \sqrt{\frac{Q z}{g n K}}
$$

式中 $\mathrm{g}$ 一地球重力加速度, $\mathrm{m} / \mathrm{s} 2$; 
$\mathrm{n}$ 一罐道钢丝绳的数目;

$\mathrm{k}$ 一钢丝绳罐道的横向刚度, $\mathrm{N} / \mathrm{m}$;

$\mathrm{Qz}$ 一提升钢丝绳的终端载荷, $\mathrm{kg}$ 。

则提升容器在一个摆动周期内的距离为:

$$
R=v T=2 \pi v \sqrt{\frac{Q z}{g n K}}
$$

其中 $\mathrm{Qz}$ 一提升容器自重, 载重和部分尾绳的重量的总和, $\mathrm{kg}$; $\mathrm{v}$ 一提升容器在某高度的速度, $\mathrm{m} / \mathrm{s}$ 。 多绳提升系统中提升容器的摆动路径如图 2 所示。

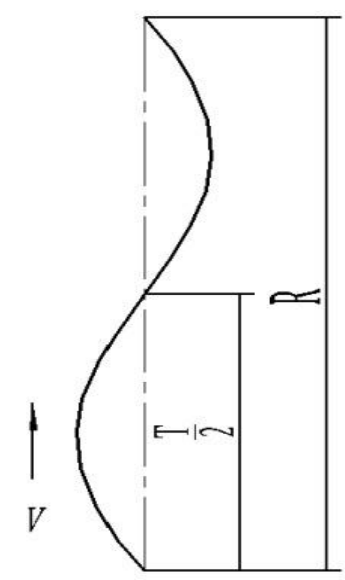

图 2 提升容器摆动距离与周期坐标关系

提升容器横向摆动量 $\mathrm{U}$ 的计算公式[11]为

$$
\begin{array}{ll}
U=-0.0144 R^{2}+1.999 R+9.86 & (\mathrm{R}<70) \\
U=0.00095 R^{2}+0.1415 R+64.4 & (\mathrm{R} \geq 70)
\end{array}
$$

\section{2 罐道绳横向刚度分析}

钢丝绳的横向刚度指引起罐道绳单位偏摆量所需横向力的大小, 对液压式钢丝绳罐道进行受力分析, 如图 3 所示。液压装置对罐道绳施加的张紧力为 $\mathrm{Q}, \mathrm{N}$; 罐道绳的单位质量为 $\mathrm{p}, \mathrm{Kg} / \mathrm{m}$; 罐道绳总长为 $\mathrm{L}, \mathrm{m}$; 罐道绳在 $\mathrm{h}$ 处受到提升容器给它的一个横向力 $\mathrm{F}$ 作用时, 它的位移量为 $\mathrm{s}$; 罐道绳上的张力分别为 $\mathrm{N}_{1}$ 和 $\mathrm{N}_{2}$; 张力与坚直方向夹角为 $\mathrm{a}$ 和 $\mathrm{b}$ 。

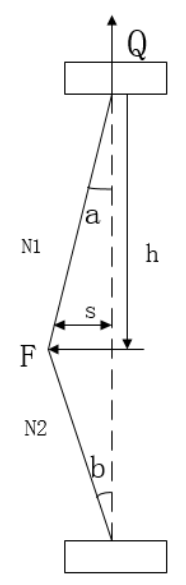

图 3 罐道绳受力示意图 
根据罐道绳受力分析图, 应用牛顿第二定律可得在水平方向有平衡公式:

F- $\mathrm{N}_{1} \operatorname{sina}-\mathrm{N}_{2} \operatorname{sinb}=0$

式中， N1 $=(\mathrm{Q}-\rho \mathrm{h}) / \cos \mathrm{,}, \mathrm{N} 2=(\mathrm{T}-\rho \mathrm{h}) / \operatorname{cosb}$

在坚直方向有平衡方程:

$\mathrm{F}=\mathrm{N}_{1} \sin \mathrm{a}+\mathrm{N}_{2} \sin \mathrm{b}=(\mathrm{Q}-\mathrm{ph}) \tan \mathrm{a}+(\mathrm{Q}-\mathrm{ph}) \tan \mathrm{b}=\frac{(Q-p h)}{h(L-h)} y$

则可得罐道绳横向刚度公式为:

$\mathrm{K}=\mathrm{F} / \mathrm{y}=\frac{(Q-p h) L}{h(L-h)}$

根据上面的计算公式，可以得到罐道绳在不同位置处的横向刚度变化曲线。取罐道绳绳长为 $760 \mathrm{~m}$, 罐 道绳单位质量为: $9.6 \mathrm{Kg} / \mathrm{m}$, 绳上张紧力为 $250 \mathrm{KN}$, 则可得到液压式张紧方式下横向刚度随运行高度变化曲 线图, 如图 4 所示:

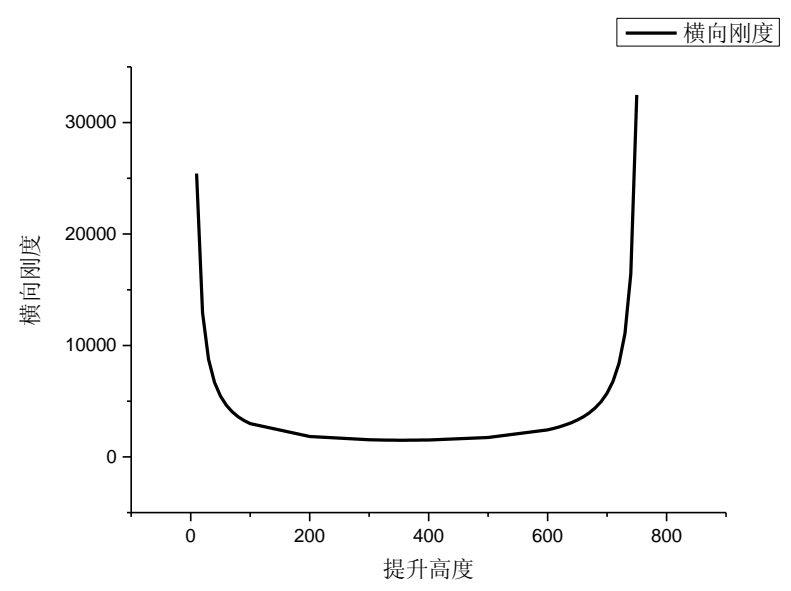

图 4 横向刚度随提升高度变化曲线

由图 4 可以看出, 罐道绳的横向刚度随着提升高度的变化而变化, 且在罐道绳两端的横向刚度值比较 大，在罐道绳中间段比较小。

\section{3 在提升系统不同参数下提升容器偏摆量计算分析}

表 1 多绳摩擦提升系统样机的主要技术参数

\begin{tabular}{cllclcc}
\hline $\begin{array}{l}\text { 提升钢丝绳 } \\
\text { 终端载荷 }\end{array}$ & $\begin{array}{l}\text { 最大提升速 } \\
\text { 度 }\end{array}$ & $\begin{array}{l}\text { 提升钢丝绳 } \\
\text { 单位质量 }\end{array}$ & $\begin{array}{l}\text { 罐道绳长 } \\
100 \mathrm{t}\end{array}$ & $\begin{array}{l}\text { 罐道钢丝绳 } \\
\text { 单位质量 }\end{array}$ & $\begin{array}{l}\text { 提升加速度 } \\
\text { 罐道绳数目 }\end{array}$ \\
\hline $10.4 \mathrm{~m} / \mathrm{s}$ & $4.94 \mathrm{~kg} / \mathrm{m}$ & $760 \mathrm{~m}$ & $9.6 \mathrm{~kg} / \mathrm{m}$ & $0.5 \mathrm{~m}^{2} / \mathrm{s}$ & 4 \\
\hline
\end{tabular}

为了验证罐道绳张紧力, 提升钢丝绳终端载荷、提升容器提升速度对提升容器在不同高度处横向偏摆 量的变化状况, 选用了罐道绳张紧力分别为 $250 \mathrm{KN}$ 和 $100 \mathrm{KN}$ 两种情况, 提升钢丝绳终端载荷分别为 $80 \mathrm{t}$ 和 $100 \mathrm{t}$ 两种情况, 最大提升速度为 $10.4 \mathrm{~m} / \mathrm{s}$ 和 $8 \mathrm{~m} / \mathrm{s}$ 两种情况。作出在不同特性参数下提升容器在不同上升 高度处的横向偏摆量对比图。 


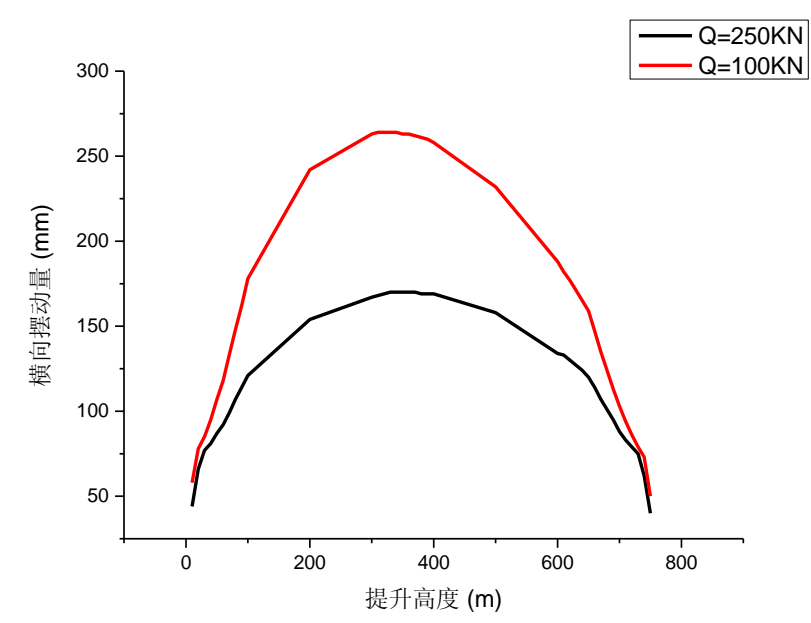

图 5 在罐道绳不同张紧力下提升容器的横向偏摆量

由图 5 可以看出提升容器在罐道绳中间部位提升容器的横向偏移量大于罐道绳两端; 罐道绳中间偏下 位置 $370 \mathrm{~m}$ 位置横向摆动量达到最大值; 罐道绳张紧力为 $250 \mathrm{KN}$ 下, 提升容器的横向偏摆量总大于罐道绳 张紧力为 $100 \mathrm{KN}$ 状态下。这是因为罐道绳中间的横向刚度随着提升容器上升逐渐减小, 减小到最小值之后 逐渐增加; 罐道绳自重导致罐道绳任意位置的张紧力不同, 罐道绳的最小横向刚度在中间偏下位置; 罐道 钢丝绳在任意位置的横向刚度随这张紧力的增大而增大。

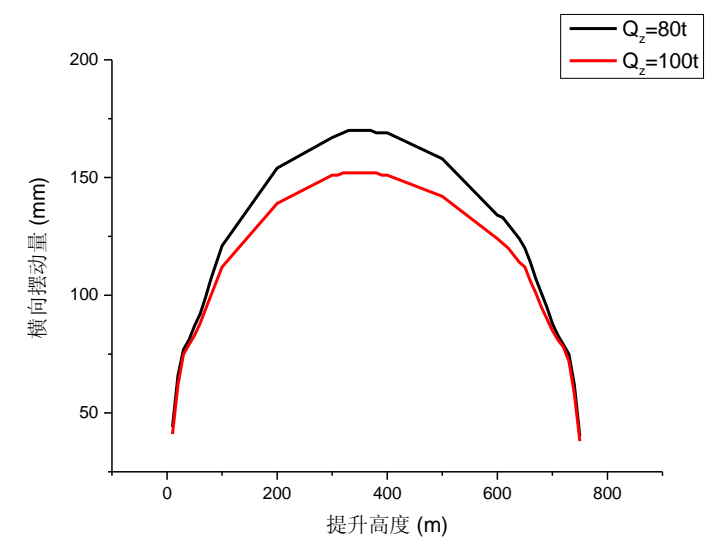

图 6 不同提升钢丝绳终端载荷下提升容器的横向摆动量

由图 6 可以看出提升钢丝绳终端载荷在罐道绳两端对提升容器的偏摆量影响较小，在提升中段 $200 \mathrm{~m}-600 \mathrm{~m}$ 时, 提升钢丝绳终端载荷 $\mathrm{Q}=80 \mathrm{t}$ 下提升容器的横向偏移量大于 $\mathrm{Q}=100 \mathrm{t}$ 状态下。因为在罐道绳两 端, 提升速度不高, 终端载荷惯性小, 提升容器横向偏摆量小, 主要是罐道绳的张紧力影响提升容器的偏 摆量。在罐道绳中段, 速度达到最大速度且罐道绳刚度减小, 提升钢丝绳终端载荷重的稳定性越高。 


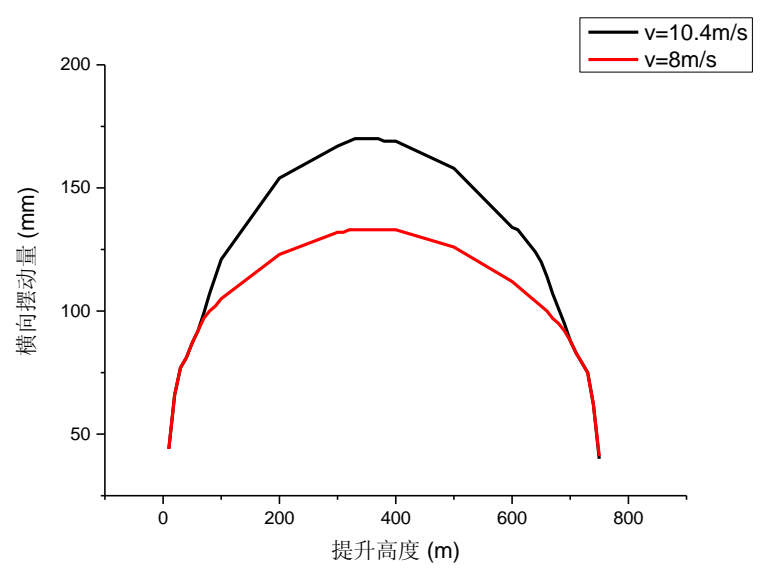

图 7 不同提升速度下提升容器横向偏摆量

图 7 可以看出, 在提升容器加减速阶段, 提升容器偏摆量的变化相同, 当提升容器达到最大提升速度 时, 最大提升速度 $\mathrm{V}=10.4 \mathrm{~m} / \mathrm{s}$ 状态下提升容器的横向偏摆量大于 $\mathrm{V}=8 \mathrm{~m} / \mathrm{s}$ 状态下。因为在罐道绳中段, 罐 道绳横向刚度减小, 速度越大提升系统的振动动能增加, 提升容器偏摆越明显。

\section{4 结论}

(1) 柔性约束下罐道绳的横向刚度与提升容器的横向偏摆量存在一定的反比例关系。在满足罐道绳破 断力前提下, 增大罐道绳上的张紧力, 能够增大罐道绳的横向刚度, 有效减小提升容器的横向偏摆量。

（2）提升容器运行到罐道绳中段, 不提升速度和提升钢丝绳终端载荷对提升容器偏摆量影响较大, 而 在起始和终点段影响较小。考虑提升钢丝绳最大承受载重和自重的前提下, 随着提升钢丝绳终端载荷的增 加, 提升容器横向偏摆量显著减少; 考虑提升系统的稳定性, 在罐道绳中段矿井提升容器的最大提升速度 控制在一定范围内。

\section{5 致谢}

\section{Acknowledgements}

The authors gratefully thank the National Key Basic Research Program of China(No. 2014CB049401), the Project of Basic and Advanced Technology Research of Henan Province of China (No. 132300410001) and the Program for Innovative Research Team (in Science and Technology) in University of Henan Province (No. 15IRTSTHN008).

\section{参考文献:}

[1] 包继虎, 张鹏, 朱昌明. 变长度柔性提升系统钢丝绳横向振动建模及分析 [J]. 上海交通大学学报, 2012. 46(3): $341-345$.

[2] 刘义, 杨芳, 夏长高, 李济顺. 摩擦提升机的横向振动特性[J]. 江苏大学学报, 2016. 37(5): 518-524.

[3] 吴娟, 寇子明, 梁敏. 摩擦提升系统钢丝绳横向动力学分析[J].振动与冲击, 2016, 02: 184-188.

[4] Kaczmarczyk S, Ostachowica W.Transient vibration phenomena in deep mine hoisting cables.Part 1: Mathematical model [J]. Journal of Sound and Vibration, 2003, 262: 219-244.

[5] Kaczmarczyk, Ostachowicz W.Transient vibration phenomena in deep mine hoisting cables.Part 2: Numerical simulation of the dynamic response [J]. Journal of Sound and Vibration, 2003, 262: 245-289.

[6] 李玉瑾. 多绳摩擦轮提升系统的动力学研究与设计[J]. 煤炭工程, 2003, 09: 6-9.

[7] 曹国华. 矿井提升钢丝绳装载冲击动力学行为研究[D]. 徐州: 中国矿业大学, 2009.

[8] 潘英. 提升钢丝绳中最大动张力的研究 [J]. 煤炭学报, 2000, 25(S1) : 138-141

[9] Ostapenko V A. Exact solution of the problem for dynamic field of displacements in rods of variable length[J].Arch Appl 
Mech, 2007, 77: 313-324

[10] 陈锡章. 钢丝绳罐道提升容器的摆动[J]. 煤炭科学技术, 1985, 02: 23-26+63

[11] 姜克城. 有关钢丝绳罐道计算方法的探讨[J]. 煤炭设计, 1991, 02: 26-30.

\section{References:}

[1] Bao Jihu, Zhang Peng, Zhu Changming. Modeling and Analysis on Transverse Vibration of Steel Wire Rope in Lifting System with Variable Length [J].Journal of Shanghai Jiaotong University,2012.46(3):341-345.

[2] Liu Yi, Yang Fan, Xia Changgao, Li Jishun. The Transverse Vibration Characteristics of Friction Hoist [J].Journal of Jiangsu University, 2016. 37(5):518-524.

[3] Wu Juan, Kou Ziming, Liang Min. Lateral Dynamics Analysis of Wire Rope Friction Hoisting System [J].Journal of Vibration and Shock, 2016, 02: 184-188.

[4] Kaczmarczyk S, Ostachowica W. Transient vibration phenomena in deep mine hoisting cables.Part 1: Mathematical model [J]. Journal of Sound and Vibration, 2003, 262: 219-244.

[5] Kaczmarczyk, Ostachowicz W. Transient vibration phenomena in deep mine hoisting cables. Part 2: Numerical simulation of the dynamic response [J]. Journal of Sound and Vibration, 2003, 262: 245-289.

[6] Li Yujin.Dynamics of Multi - Rope Friction Hoist System Research and Design [J].Coal Engineering, 2003, 09: 6-9.

[7] Cao Guohua. Study on Behavior of Load Impact Dynamics of Steel Rope in Mine [D].Xuzhou: China University of Mining and Technology, 2009.

[8] Pan Ying. Study on Maximum Hoisting Rope Dynamic Tension [J].Journal of China Coal Society,2000,25(S1):138-141

[9] Ostapenko V A. Exact solution of the problem for dynamic field of displacements in rods of variable length[J].Arch Appl Mech, 2007, 77: 313-324

[10]Chen Xizhang. Swing of Hoisting Steel Rope Road [J].Coal Science and Technology, 1985,02:23-26+63

[11]Jiang Kecheng. Discussion on Calculation Method of Steel Rope Guide [J].Coal Design, 1991, 02: 26-30. 